\title{
PRUEBAS DE ENVEJECIMIENTO ACELERADO PARA DETERMINAR VIGOR DE SEMILLAS DE Prosopis alba DE TRES PROCEDENCIAS GEOGRÁFICAS
}

\author{
Fontana, M. L. ${ }^{1,2} ;$ PereZ, V. R. ${ }^{3}$ \& Luna, C. V. ${ }^{2}$
}

\begin{abstract}
RESUMEN
Con el objeto de crear bases conceptuales para determinar la calidad de semillas de Prosopis alba a través de pruebas de envejecimiento acelerado se determinó el vigor realizando ensayos con calor húmedo (EACH), calor seco (EACS) y solución salina (EASS) en semillas de tres procedencias geográficas: Santiago del Estero, Formosa y Norte de la provincia de Salta. Se demostró significancia para la interacción Procedencia*Tratamiento y se determinó que el EACH al afectar significativamente al índice de envejecimiento es el único procedimiento aplicable a todas las procedencias. Clasificando a cada procedencia según su vigor medido a través del IE luego de la prueba de EACH: el rodal Chaqueño resulta superior a los rodales Santiagueño y Salta Norte, que son similares entre sí. La evaluación de vigor permitió distinguir las procedencias mediante cualidades no detectadas en pruebas de germinación normal en condiciones estándar.
\end{abstract}

Palabras claves: análisis de semillas, control de calidad, germinación, Mesquite.

\begin{abstract}
Accelerated aging tests for Prosopis alba to determine seed vigor of three geographical source.

In order to create conceptual basis for determining the quality of Prosopis alba's seeds through accelerated ageing tests, vigor was determinated conducting trials with moist heat (MHAA), dry heat (DHAA) and saline solution (AASS) on seeds from three geographical origins: Santiago del Estero, Formosa and north of Salta province. Significance was determined for Provenance*Treatment interaction and was determined that the MHAA is the only procedure applicable to all provenances because significantly affect the aging index (AI). According to IE measured after MHAA test, Chaco provenance showed the best performance followed by Santiago del Estero and Salta
\end{abstract}

1.- Cátedra de Silvicultura, Facultad de Ciencias Agrarias. Universidad Nacional del Nordeste. Sargento Cabral 2131. (W3402BKG) Corrientes.

Z.- Estación Experimental Agropecuaria Sáenz Peña. Instituto Nacional de Tecnología Agropecuaria.

3.- Cátedra de Silvicultura, Facultad de Recursos Naturales. Universidad Nacional de Formosa.

Email: cluna@agr.unne.edu.ar

Manuscrito recibido el 11 de diciembre de 2015 y aceptado para su publicación el 24 de junio de 2016. 


\section{L. Fontana et al.}

Norte stands, which are equal to each other. Vigor evaluation allowed the provenances to be distinguished through qualities not detected in normal germination test under standard conditions.

Key words: seed analysis, quality control, germination, Mesquite.

\section{INTRODUCCIÓN}

La calidad de semilla comprende aspectos genéticos, fitosanitarios, físicos y fisiológicos y, además, puede definirse como los atributos inherentes que determinan su potencial de germinación y sus características de crecimiento posterior (19). En este sentido, el origen de procedencia de la población de la cual se recolectan las semillas es importante ya que aunque sean de la misma especie, las semillas que se desarrollan en un gradiente latitudinal pueden mostrar características notablemente diferentes (5). Las pruebas de germinación constituyen el procedimiento más ampliamente usado para determinar calidad de semillas y, aunque sobreestiman el comportamiento de las mismas, pueden complementarse con la determinación del vigor, que brinda una respuesta que correlaciona mejor con los resultados de germinación a campo (3).

La Asociación Oficial de Análisis de Semilla (1) define a las pruebas de vigor como la herramienta cada vez más usada para la determinación del potencial fisiológico de las semillas. Los objetivos básicos de estas pruebas son: evaluar diferencias significativas en la calidad de lotes de semillas con porcentaje de germinación similar, complementar la información de las pruebas de germinación; separar lotes en diferentes niveles de vigor, relacionándolos a la emergencia de plántulas en campo, resistencia al transporte y potencial de almacenamiento. La evaluación del vigor puede realizarse mediante pruebas de estrés, las que implican la germinación de las semillas durante o después de ser sometidas a condiciones estresantes. Las mencionadas pruebas de estrés incluyen diferentes métodos tales como la prueba de frío, la prueba de germinación con frío, la prueba de estrés osmótico y la prueba de envejecimiento acelerado (EA) (20). Las pruebas de EA, basadas en el aumento del deterioro de las simientes cuando se exponen a condiciones de alta temperatura y humedad relativa por períodos de tiempo que varían según la especie, son de las más utilizadas a nivel internacional y es necesario estandarizarlas para cada especie. Una de sus deficiencias radica en que, en función de la especie y para una misma temperatura, el aumento del período de exposición proporciona ganancias en los porcentajes del contenido de agua de las simientes. Para contrarrestar este factor, se sugiere el uso de soluciones saturadas de sales $(\mathrm{NaCl}, \mathrm{KCl}$ o $\mathrm{NaBr})$ durante la realización de la prueba, con el objetivo de reducir la humedad relativa en el interior de los compartimientos individuales, con 